\title{
Supplementary Material to "Orbital control on the timing of oceanic anoxia in the Late Cretaceous"
}

\author{
Supplementary Table 1: Complete ${ }^{40} \mathrm{Ar} /{ }^{39} \mathrm{Ar}$ results
}

Sample 91-O-03, J: $0.0207634 \pm 0.0000127(1 \sigma)$, D/amu: $1.00 \pm 0.0002(1 \sigma)$, MSWD $=0.69$

Single sanidine fusions, Ages relative to 28.201 Ma Fish Canyon sanidine (Kuiper et al., 2008)

File $\quad{ }^{40} \mathrm{Ar} \quad{ }^{40} \mathrm{Ar} \quad \pm 1 \sigma_{40}{ }^{39} \mathrm{Ar} \quad \pm 1 \sigma_{39} \quad{ }^{38} \mathrm{Ar} \quad \pm 1 \sigma_{38} \quad{ }^{3 /} \mathrm{Ar} \quad \pm 1 \sigma_{37}{ }^{36} \mathrm{Ar} \quad \pm 1 \sigma_{36} \quad \%{ }^{40} \mathrm{Ar}^{*}{ }^{40} \mathrm{Ar}^{\star / 39} \mathrm{Ar}_{\mathrm{K}} \pm 2 \sigma \quad$ Age $\quad \pm 2 \sigma \quad \mathrm{K} / \mathrm{Ca} \quad$ Outliers (moles) $\left(10^{-15} \mathrm{~V}\right)\left(10^{-15} \mathrm{~V}\right)\left(10^{-15} \mathrm{~V}\right)\left(10^{-15} \mathrm{~V}\right)\left(10^{-15} \mathrm{~V}\right)\left(10^{-15} \mathrm{~V}\right)\left(10^{-15} \mathrm{~V}\right)\left(10^{-15} \mathrm{~V}\right)\left(10^{-15} \mathrm{~V}\right)\left(10^{-15} \mathrm{~V}\right)$ (Ma) (Ma)

\begin{tabular}{lllllllllllllll}
\hline BG2538 2.688E-15 0.455488 & 0.000598 & 0.173046 & 0.000268 & 0.002121 & 0.000020 & 0.001465 & 0.000167 & 0.000017 & 0.000006 & 98.87 & 2.602509 & 0.0232396 .33 & \pm 0.84 & 50.8
\end{tabular} $\begin{array}{lllllllllllllllll}\text { BG2540 1.513E-15 } & 0.256400 & 0.000567 & 0.098068 & 0.000224 & 0.001175 & 0.000019 & 0.001086 & 0.000159 & 0.000009 & 0.000006 & 99.02 & 2.588839 & 0.03975 & 95.84 & \pm 1.43 & 38.8\end{array}$ $\begin{array}{lllllllllllllllll}\text { BG2541 3.716E-15 } & 0.629596 & 0.000618 & 0.240421 & 0.000296 & 0.002879 & 0.000025 & 0.001621 & 0.000183 & 0.000021 & 0.000006 & 98.99 & 2.592404 & 0.0165395 .97 & \pm 0.60 & 63.8\end{array}$ $\begin{array}{llllllllllllllll}\text { BG2543 1.923E-15 } 0.325774 & 0.000582 & 0.125257 & 0.000245 & 0.001508 & 0.000022 & 0.000840 & 0.000168 & 0.000019 & 0.000006 & 98.24 & 2.555077 & 0.0311794 .62 & \pm 1.13 & 64.1\end{array}$ $\begin{array}{lllllllllllllllll}\text { BG2544 2.152E-15 } & 0.364594 & 0.000623 & 0.139133 & 0.000257 & 0.001626 & 0.000020 & 0.001099 & 0.000187 & 0.000010 & 0.000006 & 99.15 & 2.598308 & 0.02828 & 96.18 & \pm 1.02 & 54.4\end{array}$ $\begin{array}{lllllllllllllll}\text { BG2546 2.952E-15 } 0.500217 & 0.000615 & 0.191081 & 0.000240 & 0.002271 & 0.000024 & 0.001465 & 0.000171 & 0.000016 & 0.000006 & 99.03 & 2.592507 & 0.0211395 .97 & \pm 0.7656 .1\end{array}$

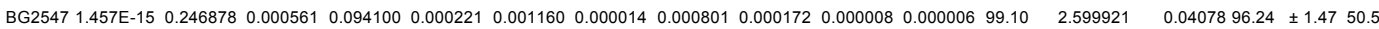

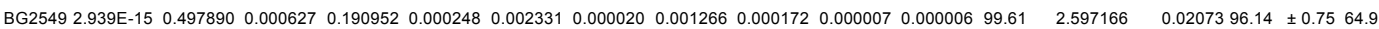
$\begin{array}{llllllllllllllll}\text { BG2550 2.535E-15 } & 0.429545 & 0.000614 & 0.163874 & 0.000244 & 0.001953 & 0.000021 & 0.001768 & 0.000169 & 0.000011 & 0.000006 & 99.23 & 2.600931 & 0.0241296 .28 & \pm 0.87 & 39.9\end{array}$

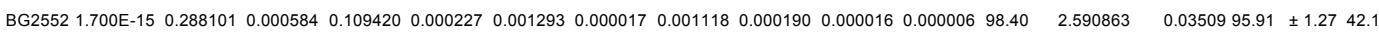
$\begin{array}{llllllllllllllll}\text { BG2555 2.418E-15 } 0.409718 & 0.000592 & 0.156706 & 0.000225 & 0.001849 & 0.000017 & 0.001257 & 0.000153 & 0.000010 & 0.000006 & 99.26 & 2.595314 & 0.02439 & 96.07 & \pm 0.88 & 53.6\end{array}$ $\begin{array}{lllllllllllllll}\text { BG2556 2.673E-15 } 0.452928 & 0.000609 & 0.173626 & 0.000257 & 0.002080 & 0.000025 & 0.001284 & 0.000162 & 0.000007 & 0.000006 & 99.55 & 2.596947 & 0.0222196 .13 & \pm 0.80 & 58.2\end{array}$

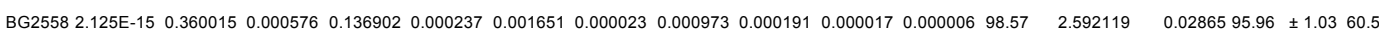
$\begin{array}{lllllllllllllll}\text { BG2559 2.424E-15 } 0.410644 & 0.000637 & 0.155942 & 0.000254 & 0.001895 & 0.000024 & 0.001459 & 0.000172 & 0.000008 & 0.000006 & 99.45 & 2.618752 & 0.0251696 .92 & \pm 0.91 & 46.0\end{array}$

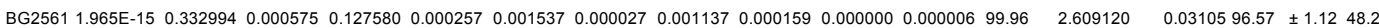

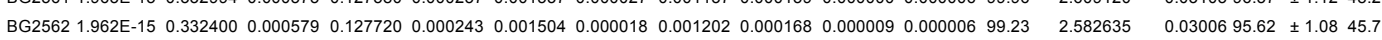

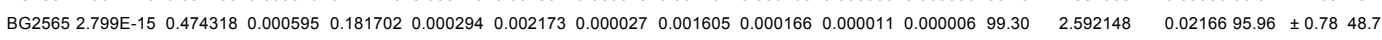
$\begin{array}{llllllllllllllll}\text { BG2567 2.447E-15 } 0.414686 & 0.000593 & 0.156068 & 0.000259 & 0.001893 & 0.000022 & 0.001004 & 0.000189 & 0.000023 & 0.000006 & 98.38 & 2.614099 & 0.0257696 .75 & \pm 0.93 & 66.9\end{array}$

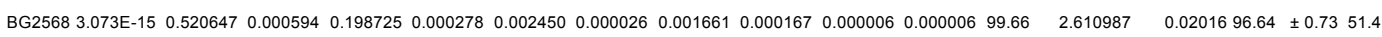
$\begin{array}{llllllllllllllll}\text { BG2570 2.071E-15 } & 0.350955 & 0.000574 & 0.134345 & 0.000246 & 0.001637 & 0.000021 & 0.000813 & 0.000171 & 0.000008 & 0.000006 & 99.35 & 2.595250 & 0.0292096 .07 & \pm 1.05 & 71.0\end{array}$ $\begin{array}{lllllllllllllllll}\text { BG2571 2.002E-15 } 0.339143 & 0.000577 & 0.129811 & 0.000240 & 0.001553 & 0.000019 & 0.000877 & 0.000149 & 0.000007 & 0.000006 & 99.37 & 2.596017 & 0.0300396 .10 & \pm 1.08 & 63.6\end{array}$ $\begin{array}{lllllllllllllll}\text { BG2573 2.735E-15 } 0.463406 & 0.000591 & 0.176698 & 0.000259 & 0.002172 & 0.000025 & 0.001557 & 0.000174 & 0.000010 & 0.000006 & 99.37 & 2.606031 & 0.0222396 .46 & \pm 0.80 & 48.8\end{array}$

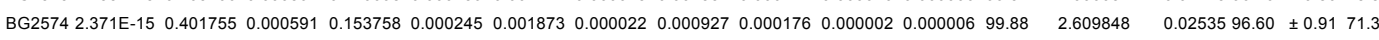

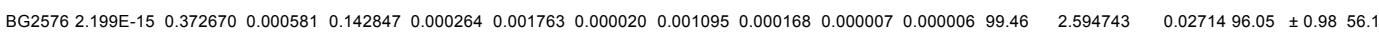

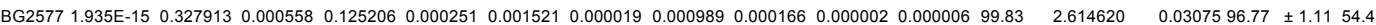
$\begin{array}{llllllllllllllll}\text { BG2579 1.732E-15 } 0.293406 & 0.000568 & 0.112215 & 0.000214 & 0.001345 & 0.000023 & 0.000902 & 0.000177 & 0.000003 & 0.000006 & 99.68 & 2.606365 & 0.03485 & 96.47 & \pm 1.26 & 53.5\end{array}$ $\begin{array}{llllllllllllllll}\text { BG2580 1.724E-15 } & 0.292050 & 0.000569 & 0.111667 & 0.000234 & 0.001335 & 0.000017 & 0.000895 & 0.000165 & 0.000004 & 0.000006 & 99.64 & 2.605889 & 0.0351196 .46 & \pm 1.27 & 53.6\end{array}$ $\begin{array}{llllllllllllll}\text { BG2582 1.892E-15 } 0.320504 & 0.000371 & 0.122576 & 0.000181 & 0.001475 & 0.000016 & 0.000906 & 0.000158 & 0.000001 & 0.000004 & 99.90 & 2.612138 & 0.0204696 .68 & \pm 0.7458 .2\end{array}$

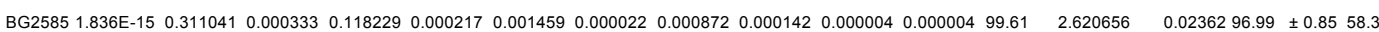
$\begin{array}{llllllllllllllll}\text { BG2588 1.557E-15 } & 0.263847 & 0.000344 & 0.100811 & 0.000137 & 0.001190 & 0.000017 & 0.000593 & 0.000144 & 0.000007 & 0.000004 & 99.23 & 2.597069 & 0.0274396 .14 & \pm 0.99 & 73.1\end{array}$

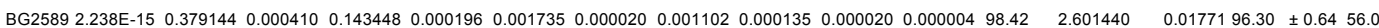
$\begin{array}{lllllllllllllllllll}\text { BG2591 1.347E-15 } 0.228257 & 0.000379 & 0.087467 & 0.000145 & 0.001030 & 0.000013 & 0.000871 & 0.000137 & 0.000014 & 0.000004 & 98.21 & 2.562801 & 0.0276694 .90 & \pm 1.00 & 43.2\end{array}$ $\begin{array}{lllllllllllllllll}\text { BG2592 1.699E-15 } & 0.287944 & 0.000362 & 0.110065 & 0.000164 & 0.001350 & 0.000017 & 0.001010 & 0.000141 & 0.000007 & 0.000004 & 99.27 & 2.596988 & 0.02143 & 96.14 & \pm 0.77 & 46.8\end{array}$

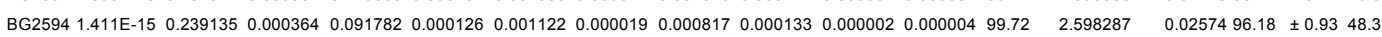
BG2595 1.337E-15 $0.2265150 .0003470 .0863780 .0001430 .0010560 .0000190 .0004870 .0001420 .0000030 .00000499 .65 \quad 2.613206 \quad 0.0270396 .72 \pm 0.9776 .2$ $\begin{array}{lllllllllllllll}\text { BG2597 1.697E-15 } 0.287521 & 0.000348 & 0.109701 & 0.000161 & 0.001331 & 0.000021 & 0.000811 & 0.000139 & 0.000019 & 0.000003 & 98.05 & 2.569814 & 0.0211195 .16 & \pm 0.7658 .2\end{array}$

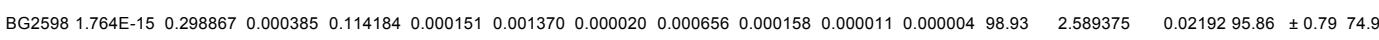
$\begin{array}{llllllllllllllll}\text { BG2600 } 1.410 \mathrm{E}-15 & 0.238883 & 0.000350 & 0.090732 & 0.000121 & 0.001113 & 0.000018 & 0.000970 & 0.000158 & 0.000021 & 0.000004 & 97.39 & 2.564202 & 0.0264494 .95 & \pm 0.95 & 40.2\end{array}$ $\begin{array}{llllllllllllllll}\text { BG2601 1.063E-15 } & 0.180108 & 0.000332 & 0.068258 & 0.000134 & 0.000847 & 0.000016 & 0.000327 & 0.000144 & 0.000015 & 0.000004 & 97.50 & 2.572580 & 0.0352695 .25 & \pm 1.27 & 89.6\end{array}$ $\begin{array}{lllllllllllllllll}\text { BG2926 3.465E-15 } & 0.587096 & 0.000442 & 0.224134 & 0.000225 & 0.002679 & 0.000023 & 0.001883 & 0.000210 & 0.000019 & 0.000003 & 99.04 & 2.594313 & 0.01123 & 96.04 & \pm 0.41 & 51.2\end{array}$

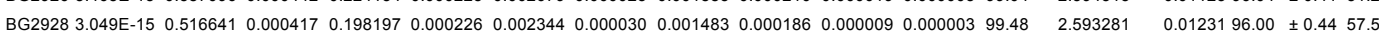
$\begin{array}{llllllllll} & \end{array}$

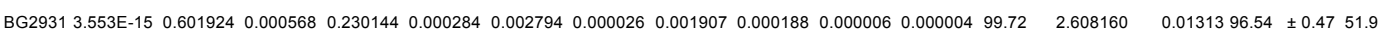

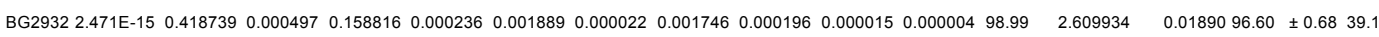
$\begin{array}{lllllllllllllllll}\text { BG2934 2.576E-15 } & 0.436471 & 0.000538 & 0.167862 & 0.000238 & 0.002053 & 0.000018 & 0.001028 & 0.000178 & 0.000007 & 0.000004 & 99.55 & 2.588466 & 0.0178995 .83 & \pm 0.65 & 70.2\end{array}$ $\begin{array}{lllllllllllllll}\text { BG2935 3.511E-15 } 0.594817 & 0.000540 & 0.215834 & 0.000254 & 0.002616 & 0.000025 & 0.001278 & 0.000184 & 0.000016 & 0.000005 & 99.18 & 2.733345 & 0.01695 & 101.05 \pm 0.61 & 72.6\end{array}$

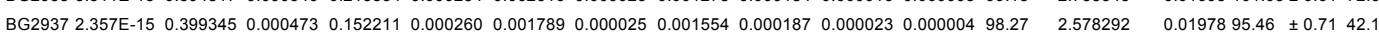
$\begin{array}{lllllllllllllll}\text { BG2938 2.430E-15 } 0.411701 & 0.000530 & 0.158137 & 0.000285 & 0.001904 & 0.000020 & 0.001058 & 0.000186 & 0.000022 & 0.000004 & 98.42 & 2.562437 & 0.0188594 .89 & \pm 0.68 & 64.3\end{array}$

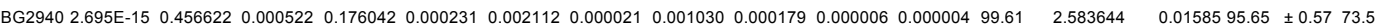
$\begin{array}{lllllllllllllll}\text { BG2941 2.960E-15 } 0.501440 & 0.000575 & 0.192708 & 0.000218 & 0.002276 & 0.000018 & 0.001395 & 0.000170 & 0.000003 & 0.000004 & 99.85 & 2.598098 & 0.0150196 .18 & \pm 0.54 & 59.4\end{array}$ $\begin{array}{llllllllllllllll}\text { BG2943 3.064E-15 } 0.519164 & 0.000535 & 0.198481 & 0.000251 & 0.002418 & 0.000027 & 0.001542 & 0.000197 & 0.000010 & 0.000004 & 99.44 & 2.600966 & 0.01495 & 96.28 & \pm 0.54 & 55.4\end{array}$ $\begin{array}{lllllllllllllllll}\text { BG2946 2.542E-15 } & 0.430646 & 0.000530 & 0.164530 & 0.000226 & 0.001992 & 0.000021 & 0.001714 & 0.000188 & 0.000009 & 0.000004 & 99.40 & 2.601626 & 0.0178596 .30 & \pm 0.64 & 41.3\end{array}$

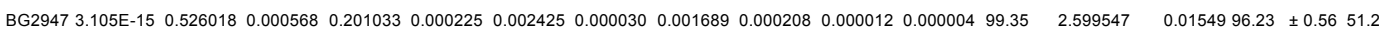

Data from neutron fluence montor crystals of Fish Canyon Sanidine (FCs)

$\begin{array}{lllllllllll}\text { Mass discrimination D/amu: } 1.001 \pm 0.0002(1 \sigma) & \text { MSWD }=0.54 \\ \text { File }{ }^{40} \mathrm{Ar} & { }^{40} \mathrm{Ar} \quad \pm 1 \sigma_{40}{ }^{39} \mathrm{Ar} \quad \pm 1 \sigma_{39} & { }^{38} \mathrm{Ar} \quad \pm 1 \sigma_{38} & { }^{37} \mathrm{Ar} & \pm 1 \sigma_{37} & { }^{36} \mathrm{Ar} & \pm 1 \sigma_{36} & { }^{*}{ }^{40} \mathrm{Ar}^{*}{ }^{40} \mathrm{Ar}^{*} / \mathrm{Ar}_{\mathrm{K}} \pm 1 \sigma & \mathrm{J} & \pm 2 \sigma & \mathrm{K} / \mathrm{Ca} \text { Outliers }\end{array}$

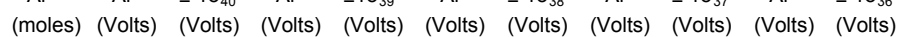

\begin{tabular}{llllllllllllllll}
\hline BF9104 2.076E-14 3.516720 & 0.035443 & 4.506578 & 0.007733 & 0.054688 & 0.000146 & 0.047081 & 0.000361 & 0.000534 & 0.000010 & 95.55 & 0.745621 & 0.00368 & 0.0208222 & \pm 0.000103 & 41.2
\end{tabular}

$\begin{array}{llllllllllllllll}\text { BF9105 3.523E-14 } 5.969541 & 0.065522 & 7.937673 & 0.007861 & 0.095540 & 0.000194 & 0.052232 & 0.000355 & 0.000098 & 0.000011 & 99.51 & 0.748368 & 0.00217 & 0.0207458 & \pm 0.000060 & 65.3\end{array}$ $\begin{array}{lllllllllllllllll}\text { BF9107 1.589E-14 } 2.692941 & 0.009360 & 3.501108 & 0.007627 & 0.042670 & 0.000133 & 0.025957 & 0.000213 & 0.000186 & 0.000009 & 97.96 & 0.753503 & 0.00470 & 0.0206044 & \pm 0.000128 & 58.0 & X\end{array}$ $\begin{array}{llllllllllllllllll}\text { BF9108 1.992E-14 } 3.374348 & 0.010170 & 4.352846 & 0.007726 & 0.053111 & 0.000156 & 0.030149 & 0.000246 & 0.000403 & 0.000011 & 96.48 & 0.747884 & 0.00388 & 0.0207592 & \pm 0.000108 & 62.1\end{array}$ $\begin{array}{lllllllllllllllll}\text { BF9110 3.659E-14 } 6.200087 & 0.023474 & 7.815288 & 0.007829 & 0.096371 & 0.000197 & 0.053106 & 0.000371 & 0.001171 & 0.000018 & 94.42 & 0.749049 & 0.00246 & 0.0207269 & \pm 0.000068 & 63.3\end{array}$

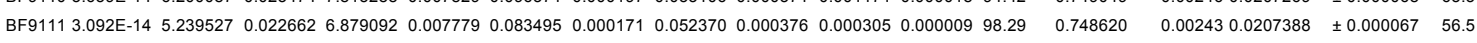
$\begin{array}{lllllllllllllllll}\text { BF9113 4.077E-14 } 6.908084 & 0.031763 & 9.150692 & 0.007918 & 0.110224 & 0.000199 & 0.061748 & 0.000436 & 0.000243 & 0.000011 & 98.96 & 0.747067 & 0.00189 & 0.0207819 & \pm 0.000053 & 63.7\end{array}$ $\begin{array}{llllllllllllllll}\text { BF9114 1.539E-14 } 2.607770 & 0.010494 & 3.410169 & 0.007620 & 0.041550 & 0.000130 & 0.023712 & 0.000198 & 0.000203 & 0.000012 & 97.70 & 0.747091 & 0.00500 & 0.0207813 & \pm 0.000139 & 61.8\end{array}$

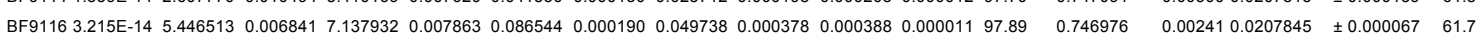

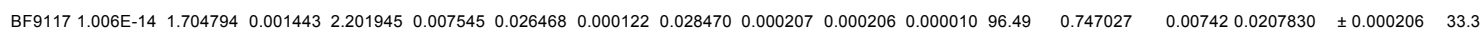




\begin{tabular}{|c|c|c|}
\hline \multicolumn{3}{|c|}{ Atmospheric argon ratios } \\
\hline${ }^{40} \mathrm{Ar} /{ }^{36} \mathrm{Ar}$ & $295.5 \pm 0.5$ & Steiger \& Jäger (1977) \\
\hline${ }^{38} \mathrm{Ar} /{ }^{36} \mathrm{Ar}$ & $0.1880 \pm 0.0003$ & Nier (1950) \\
\hline \multicolumn{3}{|c|}{ Interfering isotope production ratios } \\
\hline$\left({ }^{40} \mathrm{Ar} /{ }^{39} \mathrm{Ar}\right)_{\mathrm{K}}$ & $(5.4 \pm 1.4) \times 10^{-4}$ & Jicha and Brown (2013) \\
\hline$\left({ }^{38} \mathrm{Ar} /{ }^{39} \mathrm{Ar}\right)_{\mathrm{K}}$ & $(1.210 \pm 0.002) \times 10^{-2}$ & Jicha and Brown (2013) \\
\hline$\left({ }^{39} \mathrm{Ar} /{ }^{37} \mathrm{Ar}\right)_{\mathrm{C}}$ & $(6.95 \pm 0.09) \times 10^{-4}$ & Renne et al. (2013) \\
\hline$\left({ }^{38} \mathrm{Ar} /{ }^{37} \mathrm{Ar}\right)_{\mathrm{Ca}}$ & $(1.96 \pm 0.08) \times 10^{-5}$ & Renne et al. (2013) \\
\hline$\left({ }^{36} \mathrm{Ar} /{ }^{37} \mathrm{Ar}\right)_{\mathrm{C}}$ & $(2.65 \pm 0.022) \times 10^{-4}$ & Renne et al. (2013) \\
\hline \multicolumn{3}{|c|}{ Decay constants } \\
\hline $\mathrm{I}_{40 \mathrm{Ar}}$ & $(0.580 \pm 0.014) \times 10^{-10} a^{-1}$ & Min et al. (2000) \\
\hline $\mathrm{I}_{\mathrm{B}^{-}}$ & $(4.884 \pm 0.099) \times 10^{-10} a^{-1}$ & Min et al. (2000) \\
\hline${ }^{39} \mathrm{Ar}$ & $(2.58 \pm 0.03) \times 10^{-3} \mathrm{a}^{-1}$ & Stoenner et al. (1965) \\
\hline${ }^{37} \mathrm{Ar}$ & $(5.4300 \pm 0.0063) \times 10^{-2} a^{-1}$ & Renne \& Norman (2001) \\
\hline${ }^{36} \mathrm{Cl} \mathrm{l}$ & $(2.35 \pm 0.02) \times 10^{-6} \mathrm{a}^{-1}$ & Endt (1998) \\
\hline
\end{tabular}

All lithologic, geophysical, and stable isotope data will be made available in the Pangaea database (http://www.pangaea.de/). 